\title{
Construction delay of a penstock
}

\author{
Mohamed El Tani ${ }^{1,}$, and Fadi Hage Chehade ${ }^{2}$ \\ ${ }^{1}$ Rockgro, Po Box 11-9054, Beirut, Lebanon \\ ${ }^{2}$ LU, Dept of Civil Engineering, Faculty of Technology and Doctoral School of Science and \\ Technology, Lebanon
}

\begin{abstract}
Uncertainties are not always quantifiable and particularly those that result from a poor management. This is the case of the construction of a penstock of a hydroelectric project. The penstock is an assembly of imbricated cylindrical blocks. The steel blocks deformed during transportation and storage. Plastic deformations and buckling are undesired effects or the blocks have to be replaced. A back analysis was made considering many different transport and storage cases. The case where the blocks were laid on a rigid ground without prevention or protection is examined with an analytic model. This is followed by an analysis of the state of stresses and a validation of the model.
\end{abstract}

\section{Introduction}

The risk in geo-engineering is related to uncertainties on the project's data [1]. The observational method is used to comply with epistemic uncertainties. The increase of information with time reduces the epistemic uncertainty and the related risk is dealt with considering contingenciy measures [2] that are compensatory actions for prompt interventions. Uncertainties are not always quantifiable and particularly those that are related to a poor management with disastrous consequences.

The extension project of a hydroelectric powerplant required the construction of a new penstock of imbricated cylindrical blocks with circular cross section. The blocks are made of steel coated with a protective cover of concrete. The variability of the mechanical and geometrical properties of the blocks was assessed according to the normative [3]. Even though the construction presented no risk and should be smooth going, unconsidered uncertainties delayed the construction. The remote location of the project site combined with poorly managed block transportation and storage retarded the construction. The blocks deformed and there was a risk of buckling and plastic deformation.

Plastic deformation and buckling are not desirable and every block that undergoes such deformations cannot be used and have to be replaced. The analysis considered many storage cases, one of these is laying the cylindrical blocks on the ground without prevention and protection. This note develops an analytical formulation of the elastic behaviour of blocks that rest laterally on a sideline and deform under their own-weight. A stress analysis will be made in the elastic range up to the yield limit that should not be exceeded. The buckling is not considered in this note but is an integrant part of the case.

* Corresponding author: info@rockgro.com 


\section{Model}

The block is modelled by a cylinder tube of circular section whose length exceeds the diameter that in turn largely exceeds the thickness. The tube rests laterally on a rigid ground and is loaded by its own weight. Under these conditions, it is fully justified to assume a uniform deformation of the tube in the axial direction and to simplify the study to the deformation of the cross section that here is a circle. Fig. 1 shows a laterally laid tube, its dimensions, the cross section, applied loads, resultant forces and moment, geometric parameters and an infinitesimal element of the cross section.

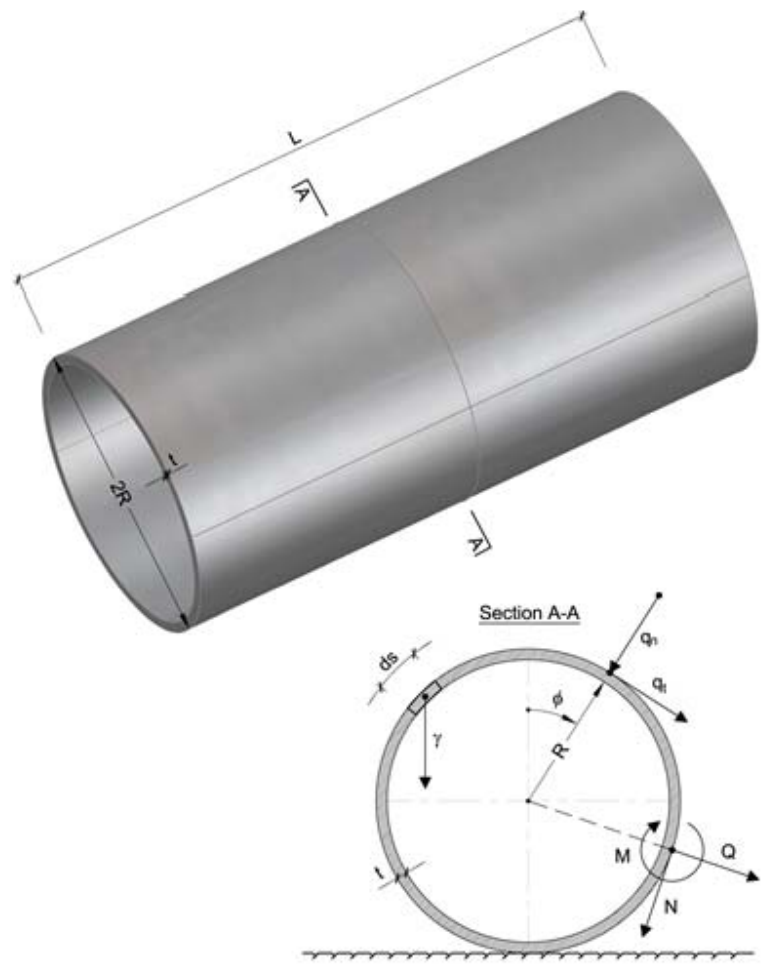

Fig. 1. Cylindrical block of circular section laid laterally.

The governing equations that are used to deduce the resultant normal and shear forces, and the moment that develop in the structure are obtained by integration. They are needed to analyse the state of stresses from which can be known if the block is irremediably damaged or still usable. Next, the deformations are obtained and are needed to compare the predictions with the measurements. Predictions and measurements have to match to validate the model and the suitability of the case. 


\subsection{Notations}

$\mathrm{N}, \mathrm{Q}$ and $\mathrm{M}$ are the resultant normal force, shear force and moment. $\mathrm{q}_{\mathrm{t}}$ and $\mathrm{q}_{\mathrm{n}}$ are the tangent and normal loads due to the self-weight. $\mathrm{v}$ and $\mathrm{w}$ are the tangent and normal displacements.

$\mathrm{R}$ and $\mathrm{t}$ are the radius of curvature and the thickness of the shell.

$\mathrm{C}$ and $\mathrm{D}$ are the stiffnesses at stretching and bending.

$\gamma$ is the specific weight.

$\mathrm{E}$ and $v$ are the elastic modulus and Poisson's ratio.

$\phi$ is the polar angle relative to the vertical axis.

$\mathrm{f}_{\mathrm{y}}$ is the nominal value of tensile strength

$\gamma_{\mathrm{M} 0}$ is the safety factor

\subsection{Governing equations}

The governing equations of a thin cylindrical shell are given in reference [4] and are the following:

$$
\begin{aligned}
& \frac{d N}{d s}+\frac{Q}{R}=-q_{t} \\
& \frac{d Q}{d s}-\frac{N}{R}=q_{n} \\
& \frac{d M}{d s}-Q=0 \\
& N=C\left(\frac{d v}{d s}+\frac{W}{R}\right) \\
& M=D\left(\frac{d}{d s} \frac{v}{R}-\frac{d^{2} w}{d^{2}}\right)
\end{aligned}
$$

In which

$$
\begin{aligned}
& \mathrm{C}=\frac{\mathrm{Et}}{\left(1-v^{2}\right)} \\
& \mathrm{D}=\frac{\mathrm{Et}^{3}}{12\left(1-v^{2}\right)}
\end{aligned}
$$

When the cross section is a circle as shown in Fig.1, the preceding equations are greatly simplified since the radius of curvature is constant and the elementary elongation in polar coordinate becomes $d s=R d \phi$. In this case, the vertical axis is a symmetry axis and it will be supposed that the horizontal displacement and the horizontal gradient of the vertical deformations are zero at $\phi=0$ and $\pi$, and at the contact with a rigid ground $w(\pi)=0$. Also, the loads on the cylindrical shell due to the self-weight are

$$
\begin{aligned}
& \mathrm{q}_{\mathrm{t}}=\gamma \mathrm{t} \sin \phi \\
& \mathrm{q}_{\mathrm{n}}=\gamma \mathrm{t} \cos \phi
\end{aligned}
$$




\subsection{Forces and moment}

The resultant normal force, shear force and moment that develop in the structure are obtained by integrating the above evolution equations and are:

$$
\begin{aligned}
& \mathrm{N}=\gamma \operatorname{Rt}(-\phi \sin \phi+\alpha \cos \phi) \\
& \mathrm{Q}=\gamma \operatorname{Rt}(\phi \cos \phi+\alpha \sin \phi) \\
& \mathrm{M}+\mathrm{RN}=\gamma \mathrm{R}^{2}(\cos \phi-1)
\end{aligned}
$$

In which

$$
\alpha=\frac{1}{2} \frac{\mathrm{R}^{2} / \mathrm{D}-1 / \mathrm{C}}{\mathrm{R}^{2} / \mathrm{D}+1 / \mathrm{C}}
$$

\subsection{Displacements}

The displacements are obtained from the material relations in Eq. 4 and 5 and the resultant forces and moments, Eq. (10) to (12) and are as follows:

$$
\begin{gathered}
\frac{\mathrm{v}}{2 \mathrm{R}^{2} \mathrm{t}}=-\frac{\lambda}{4} \phi^{2} \sin \phi-\left(\frac{3-2 \alpha}{4} \lambda+\frac{\mathrm{R}^{2}}{2 \mathrm{D}}-\frac{1}{\mathrm{C}}\right)(\phi \cos \phi-\sin \phi)+\left(\frac{\alpha}{\mathrm{C}}-\beta\right) \sin \phi-\frac{\mathrm{R}^{2}}{\mathrm{D}} \phi \\
\frac{\mathrm{w}}{2 \mathrm{R}^{2} \mathrm{t}}=\frac{\lambda}{4} \phi^{2} \cos \phi-\left(\frac{1-2 \alpha}{4} \lambda+\frac{\mathrm{R}^{2}}{2 \mathrm{D}}\right) \phi \sin \phi+\beta \cos \phi+\frac{\mathrm{R}^{2}}{\mathrm{D}} \\
\lambda=\frac{\mathrm{R}^{2}}{\mathrm{D}}+\frac{1}{\mathrm{C}} \\
\beta=\frac{\mathrm{R}^{2}}{\mathrm{D}}-\frac{\pi^{2}}{4} \lambda
\end{gathered}
$$

\section{Case study}

The geometric and mechanic properties of the blocks are a radius $\mathrm{R}=2.7 \mathrm{~m}$, thickness $\mathrm{t}=32$ $\mathrm{mm}$, elastic modulus $\mathrm{E}=210 \mathrm{GPa}$, Poisson ratio $v=0.3$ and the intrinsic weight $\gamma=80$ $\mathrm{kN} / \mathrm{m}^{3}$. The tensile strength is fy $=345 \mathrm{MPa}$ for the steel grade S355and the safety factor is $\gamma_{\mathrm{M} 0}=1.17$. Hence the limiting tensile strength that is the ratio of the nominal tensile strength to the safety factor is $295 \mathrm{MPa}$. The forces that develop in the section of a tube are shown as a function of height in Fig.2. The resultant normal force at the crown of the block is not zero and its value indicates a traction which is obtained from Eq. 10 with $\phi=0$, and is

$$
\mathrm{N}(0)=\gamma \mathrm{R} t \alpha
$$

The coefficient $\alpha$ is slightly less than one half but approaches it strongly since in this case $\mathrm{R} / \mathrm{C}$ is negligible compared to $\mathrm{R}^{3} / \mathrm{D}$ which ratio is the negligible quantity $\mathrm{t}^{2} / 12 \mathrm{R}^{2}$. Hence, the resultant traction at the crown is approximately $0.5 \gamma \mathrm{tR}$ literally and $3.5 \mathrm{kN} / \mathrm{m}$ quantitatively. The resultant traction extends over an opening of 37 degrees. Then, it 
changes sign and becomes a compressive force which extreme value is $-14.8 \mathrm{kN} / \mathrm{m}$ and attains at the ground contact $-3.5 \mathrm{kN} / \mathrm{m}$. The resultant shear is an odd function that is zero at the crown and gradually changes up to the ground contact point where it undergoes a jump that is obtained from Eq.11 as follows

$$
\Delta \mathrm{Q}=\mathrm{Q}(\pi)-\mathrm{Q}(-\pi)=-2 \pi \gamma \mathrm{Rt}
$$

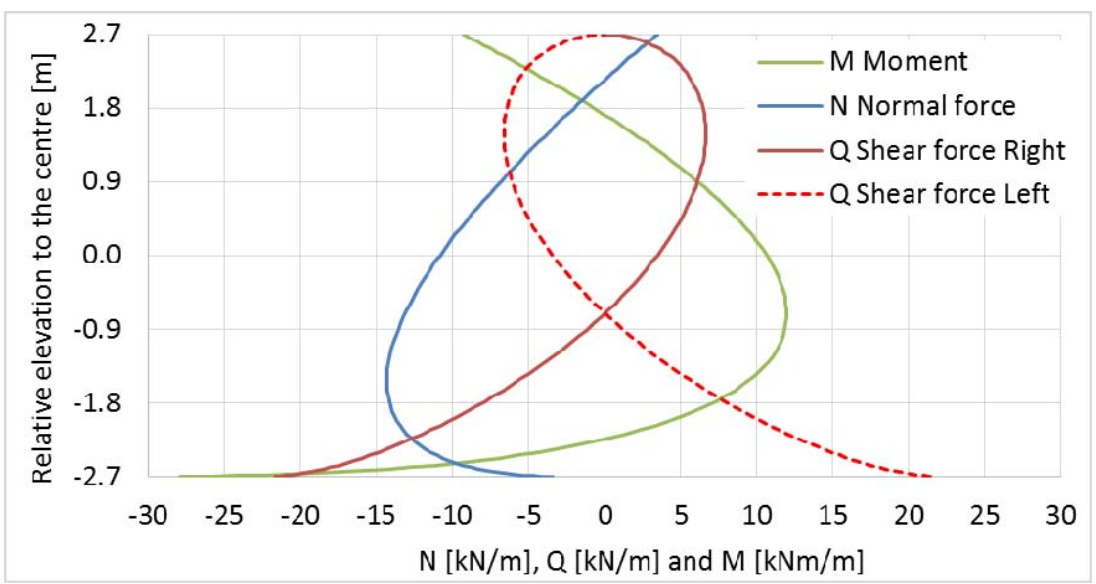

Fig. 2. Resultant forces and moment.

Fig.3 illustrates the normal stresses at the intrados and extrados of the block. They are obtained from Love's relations neglecting the curvature corrections as:

$$
\sigma=\frac{\mathrm{N}}{\mathrm{t}} \pm 6 \frac{\mathrm{M}}{\mathrm{t}^{2}}
$$

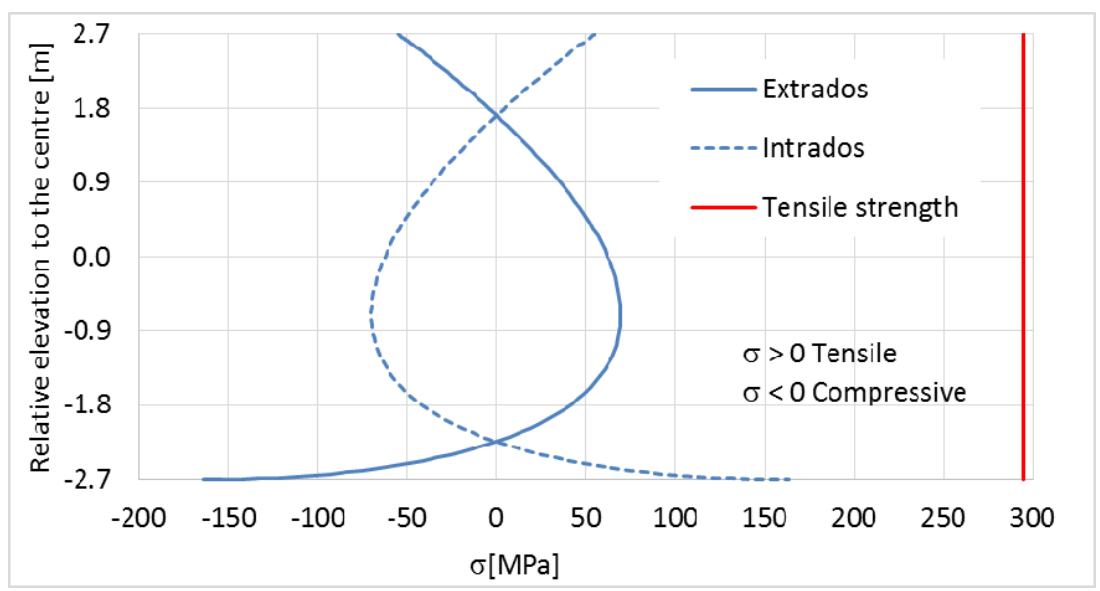

Fig. 3. Normal stresses at the intrados and extrados of the block. 
The limit tensile strength is also indicated in Fig.3. Tractions should not exceed $294 \mathrm{MPa}$ which is the ratio of the tensile strength of $345 \mathrm{MPa}$ to the safety factor 1.17. A maximum traction of $164 \mathrm{MPa}$ is attained at the ground contact point. It is inferred that the steel block does not undergo irreversible deformations since it does not plastify. The steel cylinder behaves elastically when it is loaded by its self-weight.

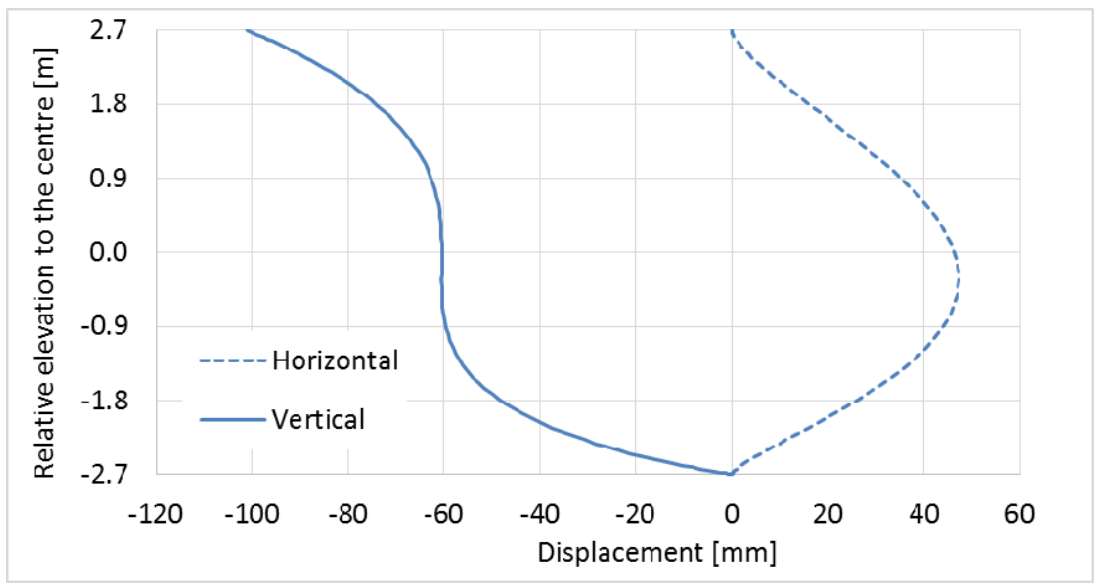

Fig. 4. Horizontal and vertical displacements.

The horizontal and vertical displacements are obtained from the radial and tangential displacements using $[\mathrm{u} \cos (\phi)+\mathrm{v} \sin (\phi)]$ and $[-\mathrm{u} \sin (\phi)+\mathrm{v} \cos (\phi)]$. The displacements on the right side are illustrated in Fig. 4; the horizontal displacement on the left side changes sign. At the crown, the horizontal displacement is zero and the vertical one $10 \mathrm{~cm}$. The vertical displacement at the mid-section is $6 \mathrm{~cm}$ and the horizontal deformation is outwards and exceeds slightly $9 \mathrm{~cm}$ or two times $4.6 \mathrm{~cm}$. The measured deformation on site varied between 2 and $6 \mathrm{~cm}$ that is less than the calculated maximal value. This validates the model as an idealistic one that needs to be relaxed considering a non-rigid ground with a larger ground-block contact area.

\section{Conclusions}

The steel blocks that were irreversibly damaged during the construction of the penstock were not damaged by laying them on the ground without protection. Other storage cases and transportation manipulations have to be analysed. The blocks that were laid without protection on a rigid ground deformed elastically and did not plastify. The calculated deformation suggests that the model needs to be relaxed.

\section{References}

1. ECN, Eurocode 7: Geotechnical design, 2004

2. A. Szavits-Nossan, Proc. XIII Conf. Geo. Eng., 2006

3. ECN, Eurocode 3: Design of steel structures, 2005

4. C.L. Dym, Introduction to the theory of shells, Pergamon, 1990 